\title{
84. Central Regulation of Thyroidal Function, especially in Relation to Nucleus Habenulae
}

\author{
S. Saito, S. Ishikawa, S. Aiba and T. Kawai \\ Department of Surgery, Gunma University Medical School
}

Current studies on pituitary-thyroidal function are focused to central regulation of this system. We have examined the role of Nucl. habenulae on the functional change of pituitary-thyroidal axis.

Lesion of Nucl. habenulae was produced by the electrocagulation. PBI and $\mathrm{PBI}^{131}$ in blood, $\mathrm{I}^{131}$ uptake of thyroid, urinary excretion of TRF, and acid phosphatase activity of pituitary were determined after the lesion. The increase of TRF excretion, $I^{131}$ uptake, PBI $^{131}$ and $\mathrm{PBI}$ levels were significant in the group of bilaterally lesioned dogs. In contrast to this, hypofunction of pituitary-thyroidal system was resulted by monolateral lesions. Acid phosphatase activity ran parallel to TRF excretion and TSH-thyroidal function. These results were thought to suggest the Thalamic-pituitary-thyroidal relationship in the mechanism of regulation of thyroidal function.

\section{Histological Studies on Pituitary and Thyroidal Gland after Long Term Injection of Thyrotrophin Releasing Factor (TRF)}

\author{
S. SaIto, C. Abe, T. Kawai and S. Ishikawa \\ Department of Surgery, Gunma University Medical School
}

We have already reported the biological property of the thyrotrophin releasing factor (TRF) to pituitary-thyroidal system; TRF produced hyperplasia and hyperfunction of thyroid by short term injection into both rats and mice. Then, we have observed histological changes of pituitary by long-term injection of TRF for 3 and 6 months.

Weights of pituitary and thyroid augmented remarkably, and beta-cell of anterior pituitary, stained by PAS and AF, increased and hypertrophyed by continued treatment of TRF. Epithelial cell of thyroid follikel, also showed proliferation and tumor-like changes, mitosis and pyknosis of nucleus were partially observed. Functional and morphological changes paralleled until six months after treatment, but after that, dissociation was seen accompanying decrease of functional situatioin. 